\title{
A simple equivalent plate model for dynamic bending stiffness of three-layer sandwich panels with shearing core
}

\author{
U. Arasan ${ }^{\mathrm{a}, \mathrm{b}, \mathrm{c}, *}$, F. Marchetti ${ }^{\mathrm{a}}$, F. Chevillotte ${ }^{\mathrm{a}, *}$, L. Jaouen $^{\mathrm{a}}$, D. \\ Chronopoulos $^{\mathrm{b}}$, E. Gourdon ${ }^{\mathrm{c}}$ \\ ${ }^{a}$ Matelys - Research Lab, F-69120 Vaulx-en-Velin, France \\ ${ }^{b}$ Institute for Aerospace Technology and The Composites Group, University of \\ Nottingham, University Park, NG7 2RD, UK \\ ${ }^{c}$ Université de Lyon, ENTPE, LTDS UMR CNRS 5513, 3 rue Maurice Audin, 69518 \\ Vaulx-en-Velin Cedex, France
}

\begin{abstract}
Equivalent or condensed plate models are being used in various industries to reduce the computation time in finite element modelling. Out of the available equivalent plate models, the model developed by J.L.Guyader in 1978 exhibits high agreement with Lamb wave theory but it requires some time for implementation. Therefore, in this paper, a simple model is proposed to quickly compute the dynamic equivalent parameters of a three-layer sandwich panel. Although the model is formulated from only four parameters, which could be easily computed via the asymptotic and transition behaviours of the sandwich panel, it is shown to be able to capture the equivalent dynamic response for the entire frequency range.
\end{abstract}

Keywords: Sandwich panels, Equivalent plate model, Flexural rigidities, Wavenumbers

\footnotetext{
*Corresponding authors:

Email addresses: arasan.uthaysuriyan@matelys.com (U. Arasan), fabien.chevillotte@matelys.com (F. Chevillotte)
} 


\section{Nomenclature}

\begin{tabular}{|cll|}
\hline Symbol & Unit & Definition \\
\hline$h$ & $\mathrm{~mm}$ & Thickness \\
$h_{t}$ & $\mathrm{~mm}$ & Total thickness \\
$\rho$ & $\mathrm{kg} \mathrm{m}^{-3}$ & Density \\
$M$ & $\mathrm{~kg} \mathrm{~m}^{-2}$ & Total mass per unit area \\
$E$ & $\mathrm{GPa}$ & Young's modulus \\
$E_{\mathrm{eq}}$ & $\mathrm{GPa}$ & Dynamic Young's modulus \\
$G$ & $\mathrm{GPa}$ & Shear modulus \\
$\nu$ & - & Poisson's ratio \\
$\eta$ & - & Loss/damping factor \\
$\eta_{\mathrm{eq}}$ & - & Dynamic loss/damping factor \\
$D$ & $\mathrm{~N} \mathrm{~m}$ & Bending stiffness \\
$D_{\mathrm{eq}}$ & $\mathrm{N} \mathrm{m}$ & Dynamic bending stiffness \\
$D_{\text {low }}$ & $\mathrm{N} \mathrm{m}$ & Low-frequency asymptote of dynamic bending stiffness \\
$D_{\mathrm{high}}$ & $\mathrm{N} \mathrm{m}$ & High-frequency asymptote of dynamic bending stiffness \\
$D_{T}$ & $\mathrm{~N} \mathrm{~m}$ & Bending stiffness at transition frequency \\
$f$ & $\mathrm{~Hz}$ & Frequency \\
$\omega$ & $\mathrm{rad} \mathrm{s}$ & Cyclic frequency \\
$f_{T}$ & $\mathrm{~Hz}$ & Transition frequency \\
$\widetilde{f_{T}}$ & $\mathrm{~Hz}$ & Approximate and simpler form of transition frequency \\
$R$ & - & Slope factor at transition frequency \\
$k$ & $\mathrm{rad} \mathrm{m}{ }^{-1}$ & Wavenumber \\
$k_{\mathrm{eq}}$ & $\mathrm{rad} \mathrm{m}{ }^{-1}$ & Equivalent bending wavenumber \\
\hline
\end{tabular}

\section{Introduction}

Multi-layered partitions have been commonly used in recent years to enhance sound comfort and noise attenuation. Sandwich composites which can exhibit high stiffness and damping with lightweight are widely employed in the transportation and building industries. This type of multi-layer is also called laminate and is often made up of three layers. One soft layer embedded between two hard skins. This kind of laminate enables to ensure a bending rigidity while increasing the dissipation by forcing the shear of the viscoelastic core. Automotive [1] and aerospace [2] industries also use sandwich structures as a passive way to reduce the structure-borne noise. Constrained layers are typically used in automobile, aircraft and railway industries to improve the damping response of the vibrating systems. In civil applications, acoustic plasterboards (with high-density core) are used to improve the sound insulation performance. 
Due to the increasing number of applications of multi-layer structures, there are many models available in the literature to predict their responses and these models are broadly categorized into three groups [3, 4]. They are 1) Equivalent Single Layer (ESL) models that describe the motion of multilayer plate as a displacement field of a single layer [5-10], 2) Layer-Wise (LW) models that describe the kinematics field in each layer [11-18] and 3) Hybrid or Zig-Zag models that make use of the advantages of the two other groups theories [19-24]. These models are applied to describe the behaviour of the multilayer. Then, from the results of these models, equivalent methodologies are applied to condense the behaviour of the multi-layer structure into an equivalent single-layer governed by frequency-dependent properties. These equivalent properties (or apparent properties [25, 26]) serve the advantage of reducing the computation time when they are used in a finite element modelling for example.

Based on the strain energy approach, a simple equivalent thin plate model was developed [27-29] (typically known as RKU model in the field) for a three-layer structure where the core layer is assumed to behave only with shear motion (which contributes for energy dissipation) and other two layers are assumed to behave only with bending motion. Due to this assumption, RKU model requires to know beforehand if each layer works in bending or shear and usually overestimates the equivalent bending stiffness and underestimates the equivalent loss factor [30]. Kurtze and Watters [31] developed a theoretical model to analyse the natural wave propagation inside a symmetric sandwich panel made of thicker core, compared to skins, based on the total impedance obtained from the bending and shear contributions of skins and core respectively. The speed of the propagating wave was computed from impedance-based dispersive relation and dynamic bending stiffness was computed from the wave speed. Recently, Zarraga et al. [32, 33] proposed a new equivalent plate model for a three-layer system based on the consideration of the low-frequency bending and shear contributions. It may be noted that this model does not account for the high-frequency bending behaviour controlled by the inner bending of the skins and does not exhibit the correct behaviour of a three-layer system at higher frequencies. Boutin and Viverge [34] used the homogenization of symmetric sandwich structure to analyse the asymptotic behaviours but this approach does not provide a dynamic model valid for the entire frequency range. Guyader and Cacciolati [35] developed an equivalent plate model (which would be referred to as Guyader model in this work hereafter) based on the previous work by Guyader and Lesueur $[19,20]$ of a hybrid model for multi-layer structures of $n$-layers. The equivalent methodology consists in assuming that the multilayer behaves as a thin plate under Love-Kirchhoff's theory. As a result, an equivalent parameter 
corresponding to the flexural rigidity of the thin plate can be identified as a function of frequency. It may be noted that, even though the equivalent plate models assume the multi-layer plate as equivalent Love-Kirchhoff plate, they account for both bending and shear motions of multi-layer plate (but not necessarily in each layer) through the frequency dependant flexural rigidity. Since Guyader model describes two anti-symmetric motions (bending and shear) in each layer, it exhibits high agreement with an exact model based on Lamb waves [36] until the frequencies where symmetric motions are no longer negligible. Marchetti et al. [37] have recently extended the Guyader model for composite structures of orthotropic layers.

Among the above mentioned analytical models available, Guyader model might be more appropriate to analyse the vibroacoustic performance of a three-layer system of isotropic materials which are commonly used across various industries. Although Guyader model performs better compared to the other equivalent plate models, it often requires some initial work for implementation as it requires many constant coefficients to be defined. Additionally, it also requires the symbolic computation of solutions from a non-linear equation which further requires solution tracing techniques to correctly capture the physically meaningful solution for the dynamic bending stiffness. Therefore, in this paper, a simple dynamic model for sandwich structure based on its asymptotic behaviours is proposed to reconstruct the dynamic response of the structure in a similar manner of the principles used for the modelling of porous media [38] or the length correction of perforated plates [39].

The present work is organised with two main sections: first, development of a simple model to find the dynamic equivalent bending stiffness of a threelayer sandwich panel is presented; then the results obtained using this new model are compared with the Guyader model for validation.

\section{Development of a simple model to compute equivalent bending stiffness of a three-layer sandwich panel}

\subsection{Dynamic behaviour of a three-layer sandwich panel}

For the theoretical development of the proposed model, Fig. 1 is used to schematically represent a generic three-layer sandwich panel of infinite extent. The $i$-th layer of the sandwich panel is assumed to be made of isotropic material with thickness $h_{i}$, Young's modulus $E_{i}$, mass density $\rho_{i}$, Poisson's ratio $\nu_{i}$ and loss/damping factor $\eta_{i}$. It is further assumed that only anti-symmetric motions (i.e, bending, shear and membrane motions) are considered for the analysis. Different configurations of layers are considered in this work using the materials (aluminium, steel, plasterboard, shear layer and polymer) listed 


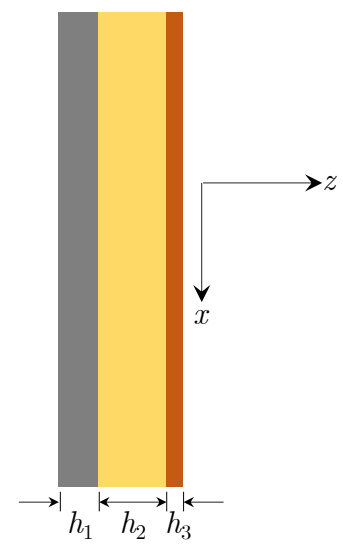

Figure 1: Schematic representation of the cross-section of a generic three-layer sandwich panel. The panel is assumed to be of infinite extent along the $x$-axis.

in Table 1. The shear layer corresponds to a layer that is sufficiently soft to exhibit shearing effects but still rigid enough to avoid compressional or dilatational effects. The asymptotic behaviours on the natural propagating wavenumber of the sandwich panel for different configurations are observed. If all three layers are of the same material, the sandwich could be considered as a homogeneous isotropic single layer. For this configuration, the natural propagating wavenumber is computed from the first-order shear deformation plate theory [5-7] and it is observed from Fig. 2a that the natural propagating wavenumber has low and high frequency asymptotes corresponding to the bending and shear motions of the panel. In case of a sandwich panel made

Table 1: Material properties of few typical elastic isotropic layers used in this paper

\begin{tabular}{cccccc}
\hline Properties & Aluminium & Steel & Plasterboard & Shear layer & Polymer \\
\hline$\rho\left(\mathrm{kg} \mathrm{m}^{-3}\right)$ & 2780 & 7800 & 700 & 200 & 580 \\
$E(\mathrm{GPa})$ & 71 & 210 & 3 & 0.1 & 0.25 \\
$\eta$ & 0.01 & 0.005 & 0.08 & 0.5 & 0.05 \\
$\nu$ & 0.3 & 0.3 & 0.22 & 0.33 & 0.33 \\
\hline
\end{tabular}

of two stiff skins ( $5 \mathrm{~mm}$ aluminium each) bonded together with a shear layer of thickness $10 \mathrm{~mm}$, the asymptotic behaviour of the natural propagating wavenumber is observed to be different from that of the isotropic single layer as shown in Fig. 2b. Furthermore, the natural propagating wavenumber of a three-layer sandwich panel could be characterized by the properties of three zones namely low-frequency, transition and high-frequency regions [40]. 


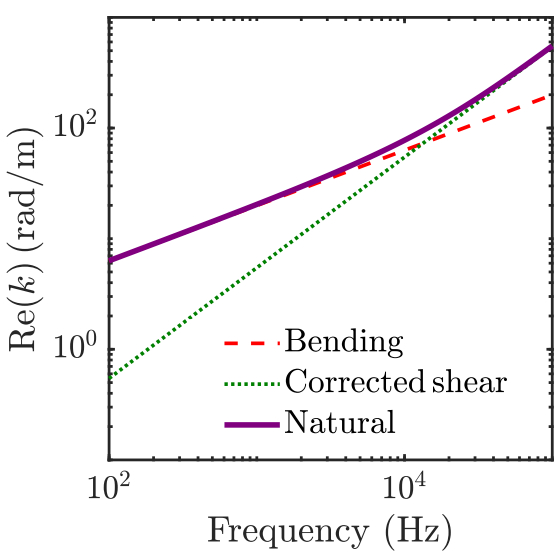

(a)

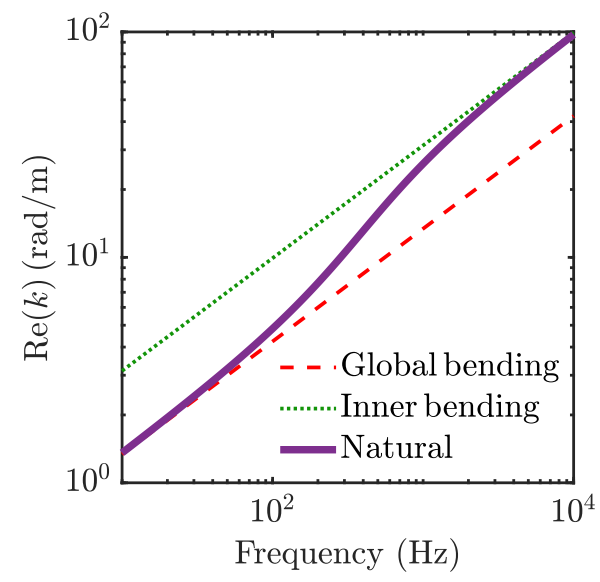

(b)

Figure 2: Natural propagating wavenumbers for (a) plasterboard of $25 \mathrm{~mm}$ (b) aluminium $(5 \mathrm{~mm}) /$ shear layer $(10 \mathrm{~mm}) /$ aluminium $(3 \mathrm{~mm})$ sandwich structure of infinite extent (material properties are listed in Table 1$)$.

The low and high frequency asymptotes correspond to the global and inner bending behaviours respectively [34]. The term "global bending" describes the bending behaviour of a three-layer sandwich panel where each layer contributes for the total bending. In case of "inner bending", only the outer layers (i.e, skins) contribute for the bending behaviour. One could note that the natural propagating wavenumber of the sandwich panel in Fig. $2 \mathrm{~b}$ is computed from the equivalent plate model by $[19,20,35]$ and this can also be computed from other models [17, 27-29] in the literature.

\subsection{Proposal of a sigmoid model}

We can observe that the equivalent bending stiffness, computed from Guyader model, has the shape of a sigmoid function for both symmetric and asymmetric sandwich structures of different configurations (Fig. 3). Thus, the goal of this paper consists in describing the equivalent parameter using this function. The sigmoid function is defined by four characteristic parameters $\left(D_{\text {low }}, D_{\text {high }}, f_{T}\right.$ and $R$ ) as shown in Fig. 4. Hence, the following expression is proposed for the equivalent bending stiffness of a sandwich structure made of isotropic layers,

$$
\log _{10} D_{\mathrm{eq}}(f)=\frac{f_{T}^{R} \log _{10} D_{\mathrm{low}}+f^{R} \log _{10} D_{\mathrm{high}}}{f^{R}+f_{T}^{R}},
$$

where $f=\omega /(2 \pi), D_{\text {low }}, D_{\text {high }}, f_{T}$ and $R$ are excitation frequency, low-frequency and high-frequency dynamic bending stiffness asymptotes, transition frequency and slope factor at transition frequency respectively. 


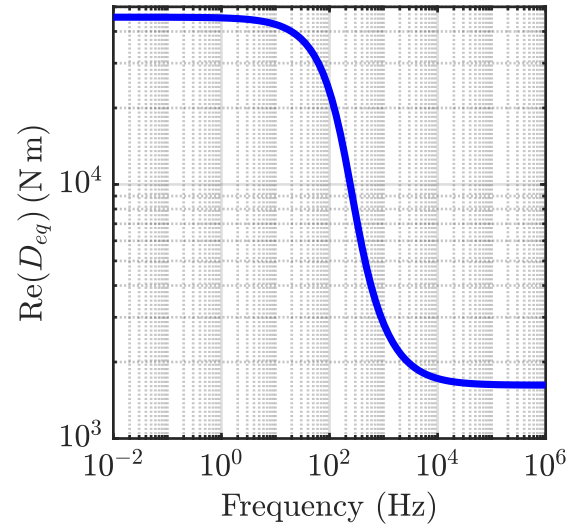

(a)

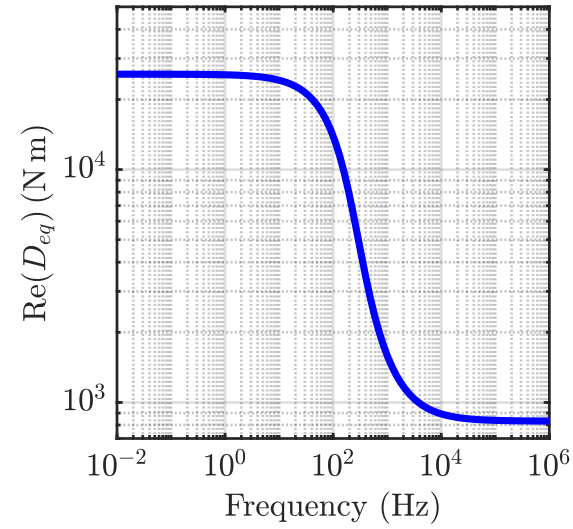

(b)

Figure 3: Equivalent bending rigidity profile obtained from Guyader equivalent plate model for (a) aluminium $(5 \mathrm{~mm}) /$ shear layer $(10 \mathrm{~mm}) /$ aluminium $(5 \mathrm{~mm})$ (b) steel $(1 \mathrm{~mm}) /$ shear layer $(10 \mathrm{~mm})$ /aluminium $(5 \mathrm{~mm})$ sandwich structures of infinite extent.

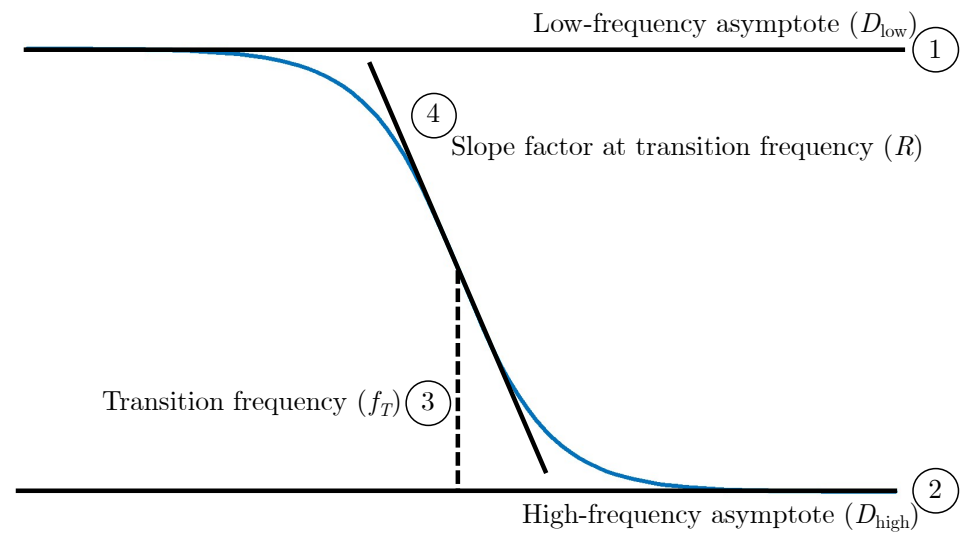

Figure 4: Schematic representation of the profile of the proposed sigmoid model and its four characteristic parameters to describe equivalent bending stiffness of a sandwich structure made of isotropic layers. 
In the following subsections, these characteristic parameters will be derived based on the relationship between the equivalent bending stiffness and material properties of the sandwich panel (Eq. (2)), given by Guyader and Cacciolati [35] to compute the equivalent bending stiffness of a multi-layer structure.

$$
A_{4} D^{3 / 2}+A_{3} D-A_{1} A_{4} D^{1 / 2}-A_{1} A_{3}+A_{2}=0,
$$

where $A_{1}=\lambda_{1}-\frac{\lambda_{5}^{2}}{\lambda_{3}}, A_{2}=\omega \sqrt{M}\left(\lambda_{4}-\frac{\lambda_{5} \lambda_{6}}{\lambda_{3}}\right)^{2}, A_{3}=\omega \sqrt{M}\left(\lambda_{2}-\frac{\lambda_{6}^{2}}{\lambda_{3}}\right)^{2}, A_{4}=$ $\lambda_{37} . M=\sum \rho_{i} h_{i}$ is the total mass per unit area and the constants $\lambda_{i}$ are defined in the Appendix A. $D_{\text {eq }}$ obtained from Eq. (2) is substituted in the following expression to find the equivalent bending wavenumber of the multi-layer structure.

$$
k_{\text {eqbending }}=\sqrt{\omega \sqrt{\frac{M}{D_{\text {eq }}}}} .
$$

Additionally, the equivalent Young's modulus, density, Poisson's ratio and loss factor are computed with the following relations.

$$
E_{\text {eq }}=\frac{12 D_{\text {eq }}\left(1-\nu_{\text {eq }}^{2}\right)}{h_{t}^{3}} ; \quad \rho_{\text {eq }}=\frac{M}{h_{t}} ; \quad \nu_{\text {eq }}=\frac{\sum \nu_{i} h_{i}}{h_{t}} ; \quad \eta_{\text {eq }}=\frac{\operatorname{Im}\left(E_{\text {eq }}\right)}{\operatorname{Re}\left(E_{\text {eq }}\right)},
$$

where $h_{t}=\sum h_{i}$ is the total thickness of the multi-layer structure.

\subsection{Low-frequency asymptote}

The lower frequency asymptote of the equivalent bending stiffness could be obtained by letting $\omega \rightarrow 0$ in the Eq. (2). This results in

$$
A_{4} D^{3 / 2}-A_{1} A_{4} D^{1 / 2}=0 \Rightarrow D=D_{\text {low }}=A_{1} .
$$

One may note that $A_{1}$ is equal to the sum of bending stiffness contribution from each layer with respect to the neutral layer position of the multi-layer structure. Assuming the top layer as the reference layer (denoted with the subscript "ref") with unit width, the transformed widths $\left(b_{i}\right)$ of the remaining layers are found with the relation [41]

$$
b_{i}=\frac{E_{i}\left(1-\nu_{\mathrm{ref}}^{2}\right)}{E_{\mathrm{ref}}\left(1-\nu_{i}^{2}\right)} .
$$

By keeping the origin of the $z$-axis at the midplane of the multi-layer plate, the neutral axis location is computed as,

$$
\bar{z}=\frac{\sum z_{i} b_{i} h_{i}}{\sum b_{i} h_{i}}
$$


Finally, $D_{\text {low }}$ is computed by adding the flexural rigidities of all the layers:

$$
D_{\text {low }}=\sum_{i=1}^{n} \frac{E_{i}}{1-\nu_{i}^{2}} \frac{\left(z_{u i}-\bar{z}\right)^{3}-\left(z_{l i}-\bar{z}\right)^{3}}{3},
$$

where $z_{i}, z_{u i}$ and $z_{l i}$ are the middle, upper and lower coordinates respectively of $i$-th layer along $z$-direction.

In case of a symmetric sandwich panel, $D_{\text {low }}$ would reduce to the form:

$$
D_{\text {low }}=D_{1}\left(8+\frac{12 h_{2}}{h_{1}}+\frac{6 h_{2}^{2}}{h_{1}^{2}}\right)+D_{2},
$$

where $D_{i}$ represents the bending stiffness of the $i$-th layer. If the core layer of the sandwich is soft compared to the skins (or outer layers), then $D_{1}, D_{3} \gg D_{2}$ which gives the following form for the low-frequency asymptote $\left(D_{\text {low }}\right)$ of the equivalent bending stiffness $\left(D_{\text {eq }}\right)$ of the sandwich panel.

$$
D_{\text {low }}=D_{1}\left(8+\frac{12 h_{2}}{h_{1}}+\frac{6 h_{2}^{2}}{h_{1}^{2}}\right) \quad \text { (for soft core). }
$$

It may be noted that this asymptotic limit can be deduced from the work by Boutin and Viverge [34] and $D_{\text {low }}$ can be understood as the result due to a phenomenon where all the layers in the sandwich panel behave as a monolithic plate governed by the global bending.

\subsection{High-frequency asymptote}

The high-frequency asymptote of the equivalent bending stiffness could be obtained by letting $\omega \rightarrow \infty$ in the Eq. (2). This results in

$$
A_{3} D-A_{1} A_{3}+A_{2}=0 \Rightarrow D=D_{\text {high }}=A_{1}-\frac{A_{2}}{A_{3}} .
$$

If the core layer of the sandwich is soft compared to the skins (or outer layers), then $D_{1}, D_{3} \gg D_{2}$ and this gives the following form for the high-frequency asymptote $\left(D_{\text {high }}\right)$ of the equivalent bending stiffness $\left(D_{\text {eq }}\right)$ of the sandwich panel:

$$
D_{\text {high }}=D_{1}+D_{3}
$$

$D_{\text {high }}$ can be understood as the result due to a phenomenon where all three layers in the sandwich panel slide on each other and the value of $D_{\text {high }}$ is governed by the intrinsic bending of each skin layers [34]. 


\subsection{Transition frequency}

Since the proposed sigmoid curve in Eq. (1) changes its sign of curvature at the geometric mean value $\left(D_{T}\right)$ of the curve (or arithmetic mean value in the log-log scale (Fig. 3)),

$$
\log _{10} D_{T}=\frac{\log _{10} D_{\text {low }}+\log _{10} D_{\text {high }}}{2} \Rightarrow D_{T}=\sqrt{D_{\text {low }} D_{\text {high }}},
$$

the transition frequency (with respect to the curvature sign of the sigmoid) is computed by substituting $D=D_{T}$ in Eq. (2) as,

$$
f_{T}=\frac{1}{2 \pi} \frac{A_{4} \sqrt[4]{D_{T}}\left(\sqrt{D_{T}}-A_{1}\right)}{A_{3}^{\prime} D_{T}+A_{2}^{\prime}-A_{1} A_{3}^{\prime}},
$$

where $A_{2}^{\prime}=\sqrt{M}\left(\lambda_{4}-\frac{\lambda_{5} \lambda_{6}}{\lambda_{3}}\right)^{2}$ and $A_{3}^{\prime}=\sqrt{M}\left(\lambda_{2}-\frac{\lambda_{6}^{2}}{\lambda_{3}}\right)^{2}$.

For softer core $\left(D_{1}, D_{3} \gg D_{2}\right)$, the transition frequency takes the following form.

$$
f_{T}=\frac{1}{2 \pi} \frac{G_{2}}{12 h_{2}} \frac{D_{\text {low }}}{\sqrt{M D_{T}}}\left(\frac{h_{1}^{2}}{D_{1}}+\frac{h_{3}^{2}}{D_{3}}\right) .
$$

In case of symmetric sandwich panel, the above expression can be written as,

$$
f_{T}=\frac{1}{2 \pi} \frac{G_{2} h_{1}^{2}}{3 h_{2}} \frac{D_{\text {low }}}{D_{\text {high }}} \frac{1}{\sqrt{M D_{T}}} .
$$

From the wavenumber analysis of the sandwich panel with a thicker core $\left(h_{2} \gg h_{1}, h_{3}\right)$, an alternate and simpler expression for the transition frequency could be derived. From Fig. 5a and 5b, it is observed that both equivalent bending (Eq. (3)) and shear wavenumbers (Eq. (17)) are equal at the transition zone when the core thickness is greater than that of the skins.

$$
k_{\text {eq }}=\omega \sqrt{\frac{M}{G_{2} h_{t}}} .
$$

On the contrary, it is also observed that this may not be valid when the core thickness is lower or equal to that of the skins. For example, from Fig. 5c, it is seen that both equivalent bending and shear wavenumbers do not have the same values at the transition zone. From the parametric study, it is further observed that the influence of the material properties of the core is less significant than the influence of the core thickness to have the equal values of equivalent bending and shear wavenumber at the transition zone. This is also complying with impedance and wave speed analysis of symmetric sandwich panel by Kurtze and Watters [31]. 


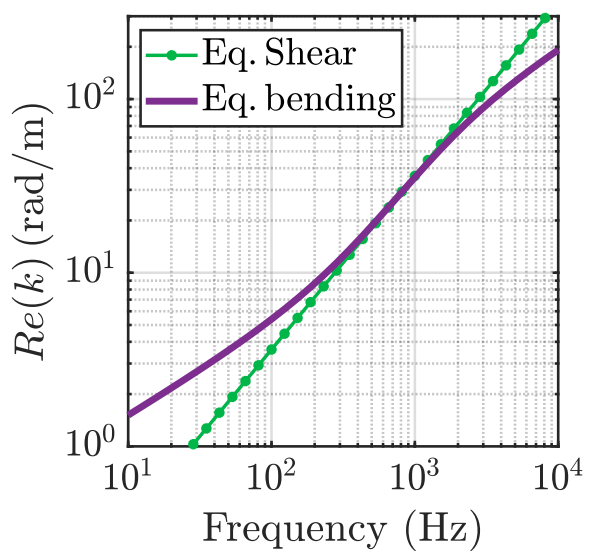

(a)

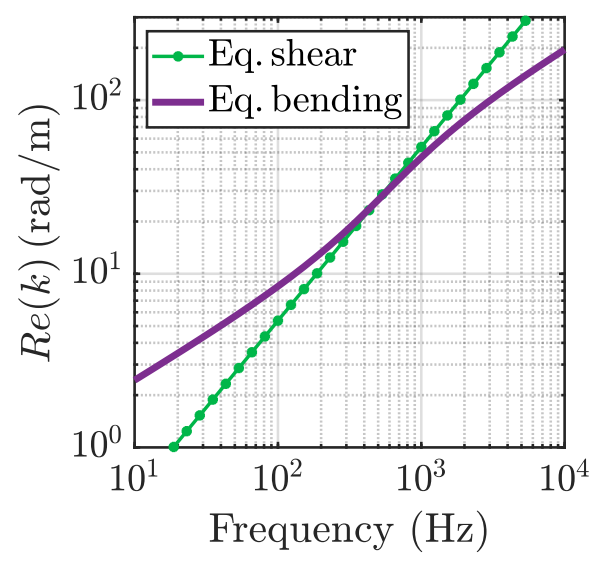

(b)

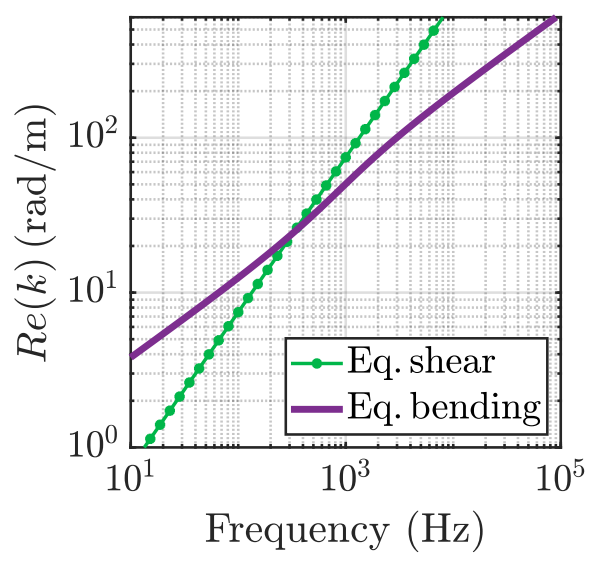

(c)

Figure 5: Equivalent bending and shear wavenumbers for a sandwich panel of infinite extent with steel skins of $1 \mathrm{~mm}$ and shear layer as core with thickness (a) $10 \mathrm{~mm}$ (b) $3 \mathrm{~mm}$ (c) $0.5 \mathrm{~mm}$. Influence of core thickness on the transition zone can be observed from these plots. 
Hence, for a thicker core, the transition frequency takes the following simpler form.

$$
k_{\text {eqbending }}=k_{\text {eq }_{\text {shear }}} \Rightarrow \sqrt{\omega_{T} \sqrt{\frac{M}{D_{T}}}}=\omega_{T} \sqrt{\frac{M}{G_{2} h_{t}}} \Rightarrow \widetilde{f_{T}}=\frac{1}{2 \pi} \frac{G_{2} h_{t}}{\sqrt{M D_{T}}} .
$$

It may be noted that, for a typical sandwich panel with a soft core, the deviation percentage of Eq. (18) from Eq. (15) would serve as an indicator on the influence of core layer in determining the transition frequency.

\subsection{Slope factor at the transition frequency}

Slope of the sigmoid curve at the transition frequency is given by (from Eq. (1)),

$$
\left.\frac{\mathrm{d} D_{\text {eq }}}{\mathrm{d} f}\right|_{f=f_{T}}=R\left[\frac{D_{T}}{4 f_{T}} \ln \left(\frac{D_{\text {high }}}{D_{\text {low }}}\right)\right]
$$

Since analytical computation of the slope,$\left.\frac{\mathrm{d} D_{\mathrm{eq}}}{\mathrm{d} f}\right|_{f=f_{T}}$, from Guyader model is cumbersome, a parametric study is preferred to compute the slope factor $(R)$. Following range of values are used for this parametric study (with symmetric case) for Young's modulus and density of the core respectively: $1 \times 10^{-5} E_{s}<E_{2}<0.1 E_{s}, 0.2 \rho_{s}<\rho_{2}<2.4 \rho_{s}$ where $E_{s}$ and $\rho_{s}$ are the reference values for Young's modulus and density for the skin respectively and Gamma distribution is considered for each parameter. As an example, the mechanical properties of aluminium could be taken for the skin to decide the range of values for the mechanical properties of the core.

From the parametric study, the envelope of the values of $R$ and its mean value are plotted in the Fig. 6 and for the practical values of core to skins thickness ratio, mean curve of $R$ is fitted into the following polynomial.

$$
R=1.16-\frac{27 \phi^{6}-52 \phi^{5}-189 \phi^{4}+275 \phi^{3}+995 \phi^{2}+291 \phi}{10^{4}},
$$

where $\phi=\log _{10}\left(\frac{h_{2}}{h_{1}+h_{3}}\right)$. It is to be noted that the parametric study is also conducted for the asymmetric case by varying the material and geometric parameters of the core and skin layers (for example, $0.5 h_{1}<h_{3}<3 h_{1}$ ). The mean curve for $R$-value obtained for asymmetric case results in maximum deviation to be lower than $1.5 \%$ to that of the symmetric case. Therefore, the polynomial fit for $R$-value given by the Eq. (20) could be applied for asymmetric configurations as well. 


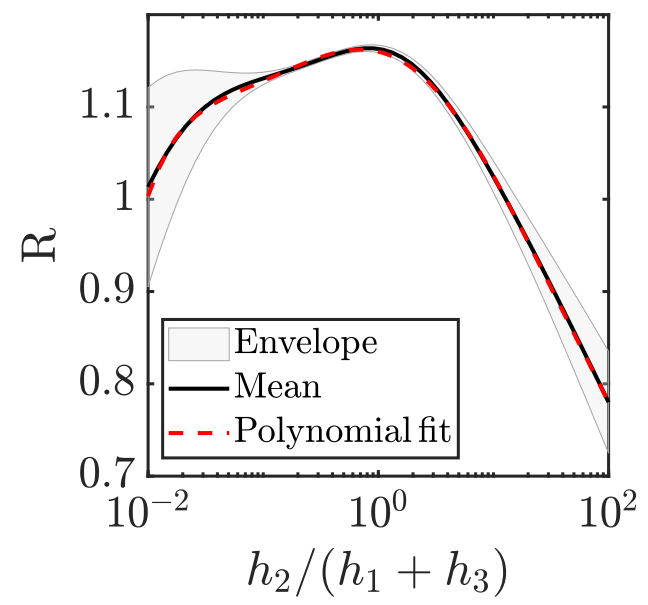

Figure 6: Envelope of $R$ and its mean against the ratio between thicknesses of core and skins.

\section{Numerical examples}

In this section, numerical examples of the proposed sigmoid model to compute equivalent bending stiffness (from Eq. (1)) of a sandwich panel and the corresponding equivalent bending wavenumber (from Eq. (3)) are presented. For the reasons mentioned and demonstrated by Ege et al. [30], Guyader model [35] is taken as a reference to compare the results of the proposed model.

In Fig. 7, for a symmetric sandwich panel made of aluminium $(5 \mathrm{~mm}) / \mathrm{soft}$ core $(10 \mathrm{~mm}) /$ aluminium $(5 \mathrm{~mm}), D_{\text {eq }}$ and $k_{\text {eqbending }}$ computed from the sigmoid model are presented for comparison, along with the transition frequency computed from Eq. (15). It can be seen from these plots that, the sigmoid model is in high agreement with the Guyader model throughout the frequency range and the observed maximum error percentage is $4.9 \%$ in comparison with Guyader model. Furthermore, it is observed from Fig. 7b that the transition frequency zone is controlled by the shear of the sandwich core as the core has double the thickness of the skin. Due to this reason, the simpler expression from Eq. (18) estimates the transition frequency as $237 \mathrm{~Hz}$ which is deviated around $14 \%$ from the value $(276 \mathrm{~Hz})$ computed by Eq. (15). One may note that this percentage of deviation would be further reduced if the thickness of the core layer is increased. 


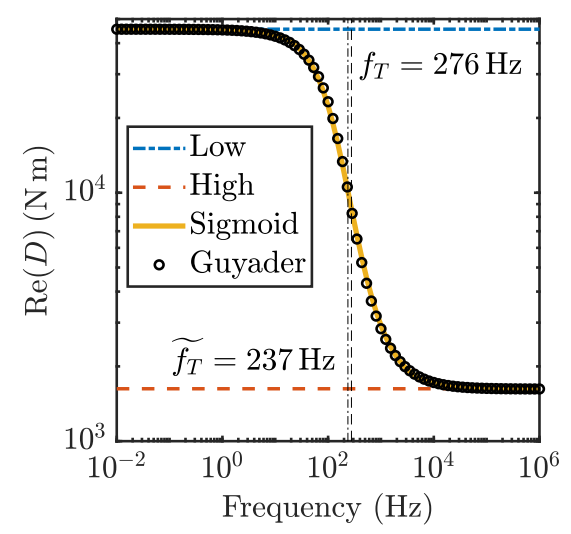

(a)

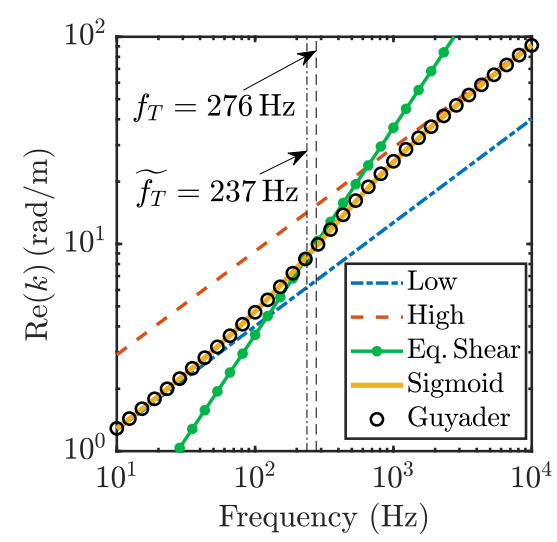

(b)

Figure 7: (a) Equivalent bending rigidity and (b) equivalent wavenumbers obtained from the proposed sigmoid model for aluminium $(5 \mathrm{~mm}) /$ shear layer $(10 \mathrm{~mm}) /$ aluminium $(5 \mathrm{~mm})$ symmetric sandwich panel of infinite extent. Guyader model is taken as reference to compare the proposed model.

In Fig. 8, for an asymmetric sandwich panel made of steel $(1 \mathrm{~mm}) / \mathrm{shear}$ layer $(0.5 \mathrm{~mm}) /$ aluminium $(5 \mathrm{~mm}), D_{\text {eq }}$ and $k_{\text {eqbending }}$ computed from the sigmoid model are presented for comparison, along with the transition frequency computed from Eq. (15). From these plots as well, it can be seen that the sigmoid model is in high agreement with the Guyader model throughout the frequency range and the observed maximum error percentage is $2.1 \%$ in comparison with Guyader model. Unlike the previous sandwich configuration, it is observed from Fig. 8b that the transition frequency zone is not controlled by the shear of the sandwich core as the core has a lesser value of thickness to that of the skins. This also reflects with a greater percentage of deviation (around 83\%) for the simpler expression of transition frequency from Eq. (18) with that of the same from Eq. (15). 


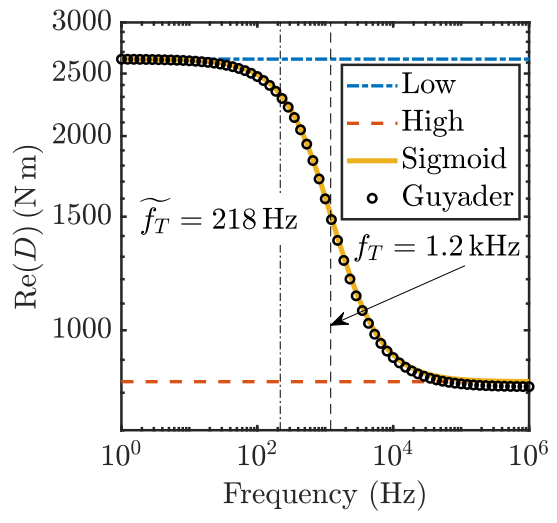

(a)

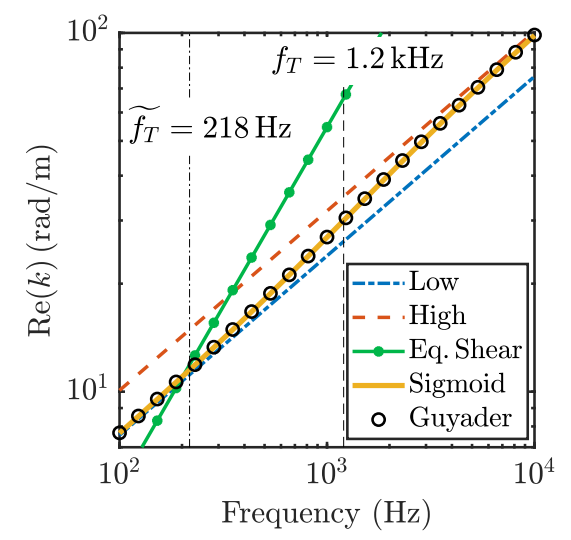

(b)

Figure 8: (a) Equivalent bending rigidity and (b) equivalent wavenumbers obtained from the proposed sigmoid model for steel $(1 \mathrm{~mm}) /$ shear layer $(0.5 \mathrm{~mm}) /$ aluminium $(5 \mathrm{~mm})$ asymmetric sandwich panel of infinite extent. Guyader model is taken as reference to compare the proposed model.

\section{Experimental validation and further observation}

In this section, the proposed model is compared with the experimental data, measured by Ege et al. [30], for the purpose of validation. A symmetric sandwich plate made of steel $(0.18 \mathrm{~mm}) /$ polymer $(0.69 \mathrm{~mm}) /$ steel $(0.18 \mathrm{~mm})$ with in-plane dimensions $300 \times 400 \mathrm{~mm}^{2}$, is considered for the experimental study and the data are measured through the contactless measurements (scanning laser vibrometer). Further, the CFAT (Corrected Force Analysis Technique) [42] methodology is used to estimate the bending stiffness of the structure. The dynamic bending stiffness can be quickly constructed, through the proposed sigmoid model, using only four parameters from Eqs. (8), (12), (15) and (20) which are substituted in Eq. (1). Finally, the equivalent Young's modulus, $E_{\text {eq }}$, is computed from Eq. (4) and compared against experimental data as shown in Fig. 9. A high agreement is observed between the estimation by equivalent plate models and the measured data which validates the applicability of the proposed model. 


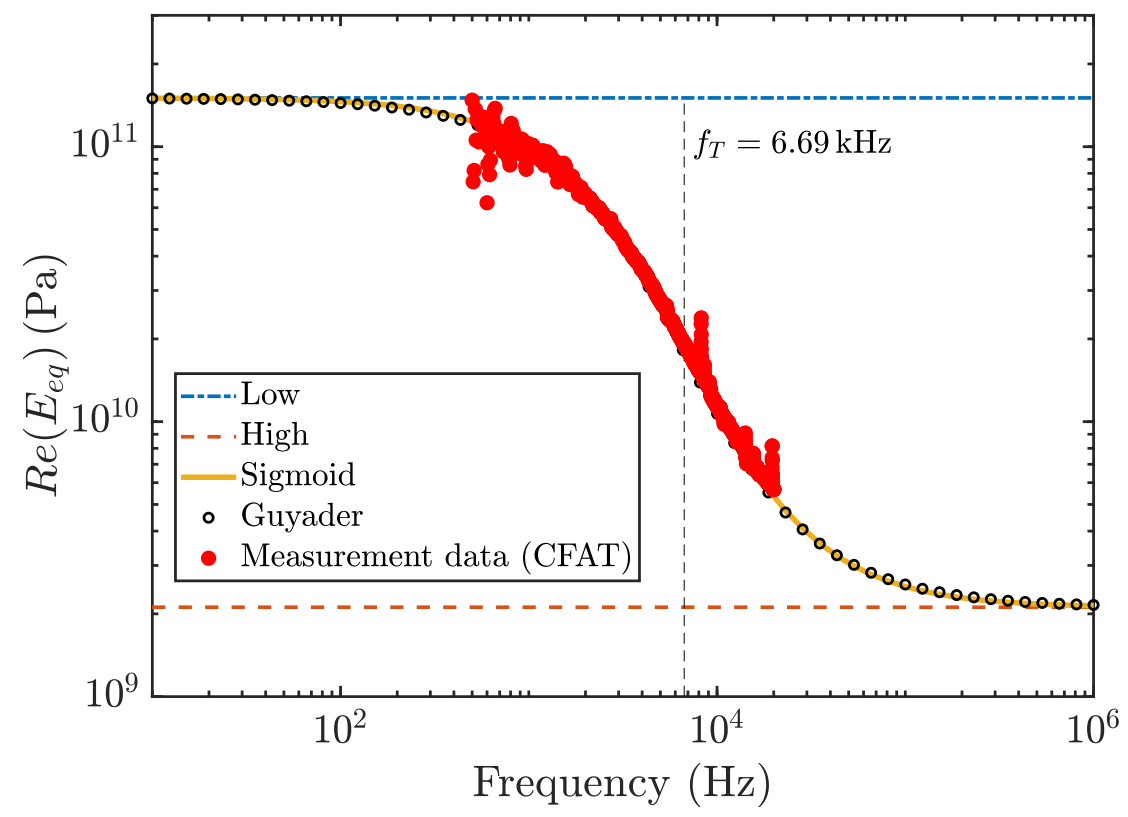

Figure 9: Comparison of equivalent plate models (proposed sigmoid model and Guyader model) with experimentally measured data of the equivalent Young's modulus for the steel $(0.18 \mathrm{~mm}) /$ polymer $(0.69 \mathrm{~mm}) /$ steel $(0.18 \mathrm{~mm})$ sandwich panel with in-plane dimensions $300 \times 400 \mathrm{~mm}^{2}$.

Through these numerical examples discussed in this work, on the implementation side, the proposed model has its advantage of using only five equations (i.e, Eqs. (1), (8), (12), (15) and (20)) whereas Guyader model requires to define seven constants and few other matrix definitions to compute the equivalent bending stiffness (see Appendix A). Further, in the Guyder model, Eq. (2) need to be solved symbolically to obtain the solutions and solution tracing techniques have to be applied to correctly capture the physically meaningful solution for $D_{\text {eq }}$. Such complexities do not present in the proposed model and it gives a straightforward solution for $D_{\text {eq }}$. On an additional note, although the proposed model focuses on reconstructing the equivalent dynamic bending stiffness values of Guyader model, it is observed from the Figs. 10 and 11 that the new model captures the equivalent dynamic loss factor of the system with the high agreement with Guyader model and experimental data. It may be noted that the noise in the measured data of Fig. 11 may be due to the instability of experimental method at low frequencies. Further, it is also observed that a slightly different Young's modulus (300 MPa) is used for the polymer by Ege et al. [30] to improve their fit on the damping loss factor. The reader may note that, although equivalent 
plate models account for both bending and shear motions of the multi-layer structures through dynamic bending stiffness, they overestimate the equivalent loss factor at high frequencies. Nevertheless, it can be corrected by the ratio between the phase and group velocities of the structure [37].

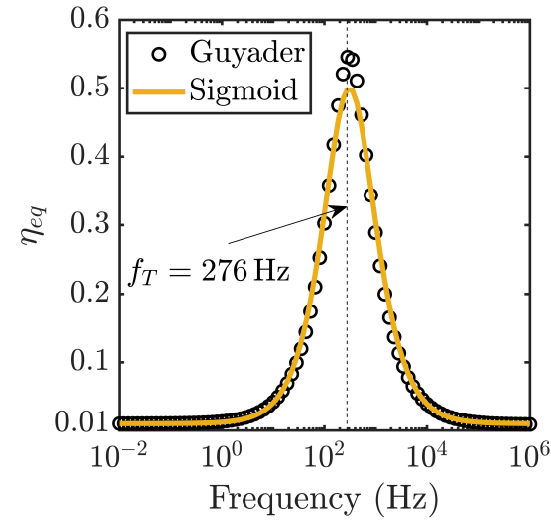

(a)

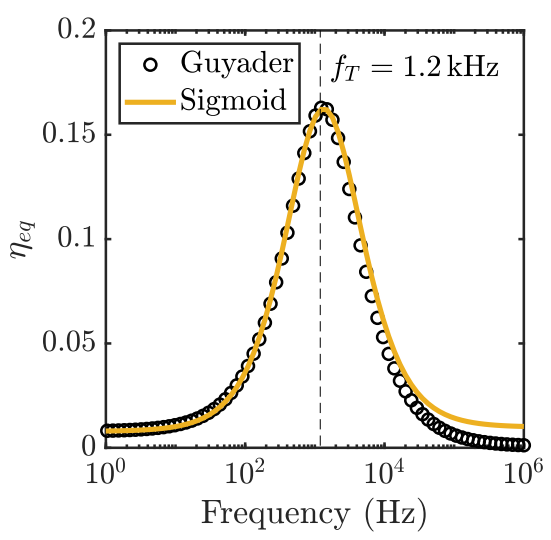

(b)

Figure 10: Equivalent loss factor for (a) symmetric aluminium $(5 \mathrm{~mm}) /$ shear layer $(10 \mathrm{~mm}) /$ aluminium $(5 \mathrm{~mm})$ (b) asymmetric steel $(1 \mathrm{~mm}) /$ shear layer $(0.5 \mathrm{~mm})$ /aluminium $(5 \mathrm{~mm})$ sandwich panel of infinite extent. Guyader model is taken as reference to compare the proposed model.

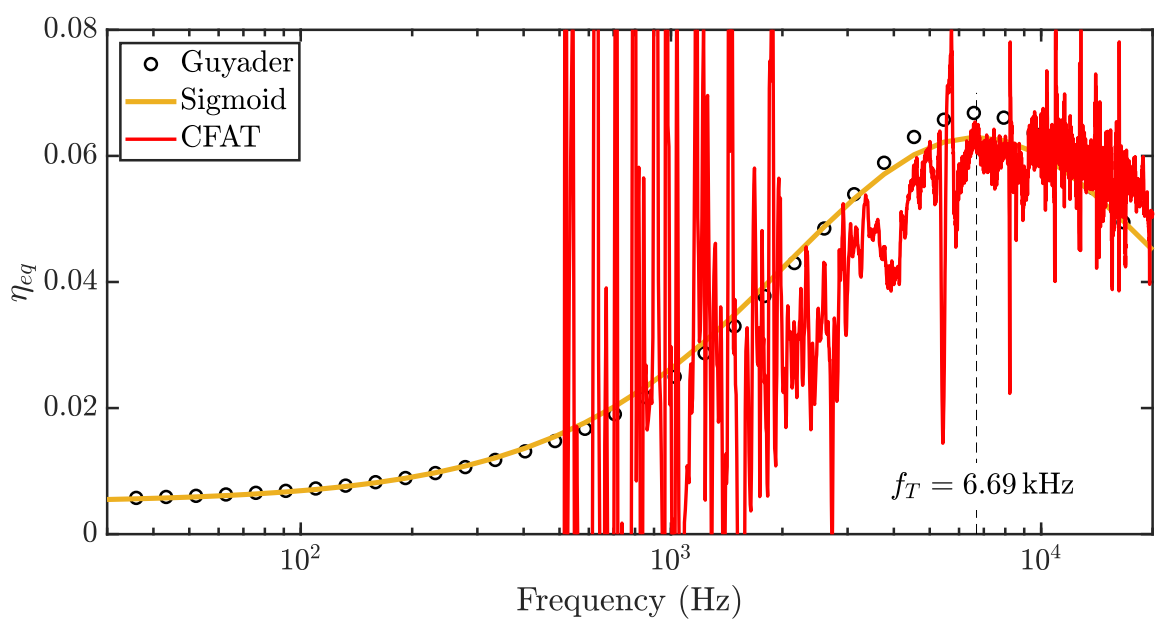

Figure 11: Comparison of equivalent plate models (proposed sigmoid model and Guyader model) with experimentally measured data of the equivalent loss factor for the steel $(0.18 \mathrm{~mm}) /$ polymer $(0.69 \mathrm{~mm}) /$ steel $(0.18 \mathrm{~mm})$ sandwich panel with in-plane dimensions $300 \times 400 \mathrm{~mm}^{2}$. 
Considering all the observations made in this work, the proposed model has its following advantages over the existing models in the literature: first, this model can be quickly implemented compared to the other equivalent plate models to compute the equivalent parameters of a three-layer sandwich panel (symmetric and asymmetric configurations); second, since the model is based on the asymptotic behaviours at different frequency regimes (low, high and transition), it can be used to understand the physics behind the response of a three-layer sandwich system at those frequency regimes and to identify the corresponding governing parameters; third, the new model will be a handy tool to optimize the layer parameters to achieve the desired damping performance of the three-layer sandwich panel due to its straightforward formulation. The reader may refer to Table 2 for the summary of all the expressions for the proposed sigmoid model. 


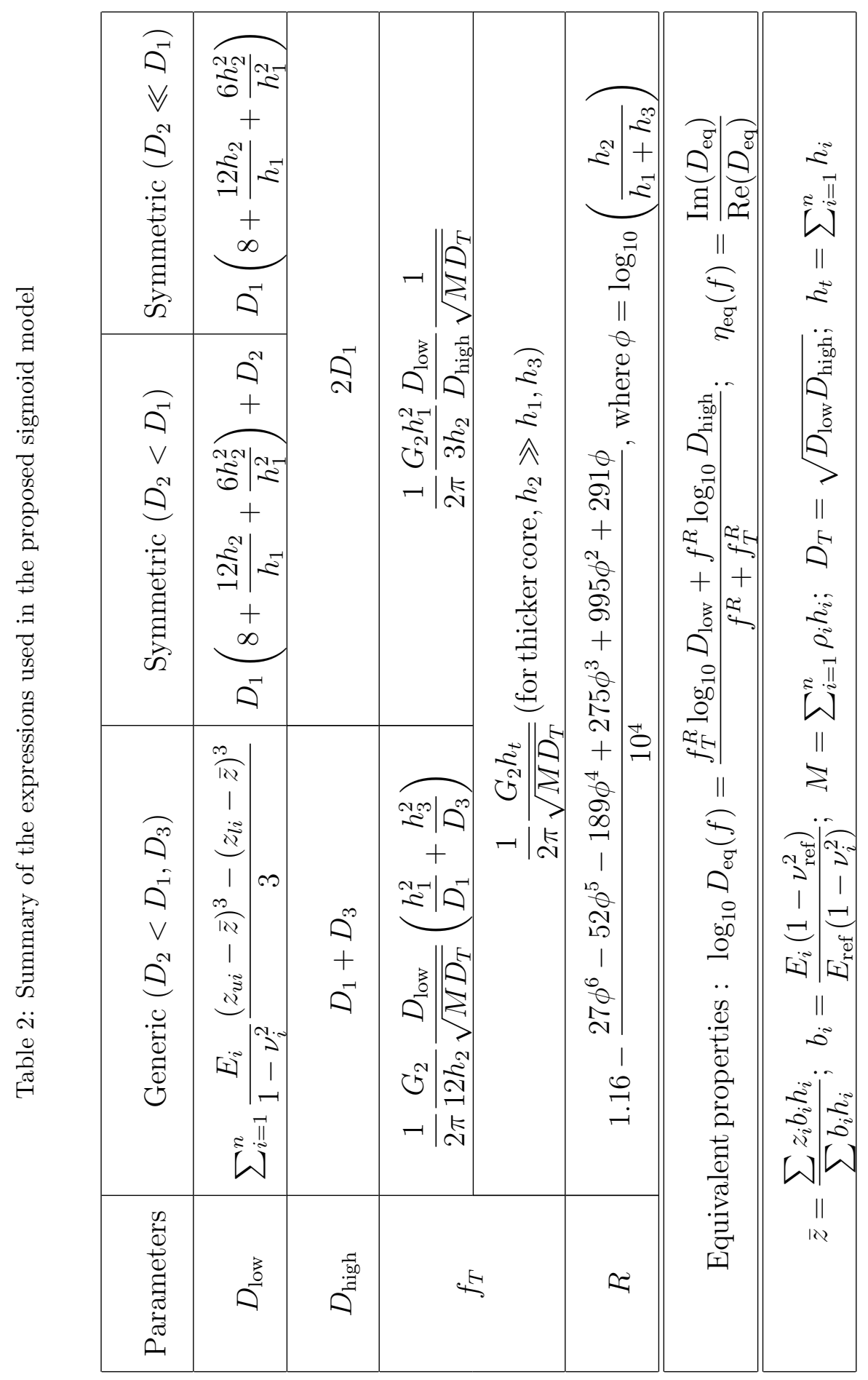




\section{Concluding remarks}

A simple equivalent plate model is proposed to compute the dynamic equivalent properties of a three-layer sandwich panel of infinite extent and made of isotropic materials. Though the formalisation of the proposed model is based on the physical behaviours at only three frequency regimes (low, high and transition), described by Fahy and Gardonio [40], it is showed that the simple model is indeed valid for the entire frequency range. In comparison with other existing equivalent plate models, the new model will be easier to implement and would serve as a tool to quickly optimize the sandwich panel parameters to obtain the desired performance.

\section{Acknowledgments}

The authors would like to gratefully acknowledge Marie-Skłodowska Curie Actions (MSCA) Project 765472 'N2N: No2Noise' for financial support.

\section{Appendix A. Definitions of constants used in Guyader model}

For $n$-layer multi-layer structure, the constants used in Guyader model [35] to compute equivalent bending stiffness are,

$$
\begin{gathered}
\lambda_{1}=\sum_{i=1}^{n} C_{11}^{i}\left(\frac{h_{i}^{3}}{12}+h_{i} \beta_{i}^{2}\right) \\
\lambda_{2}=\sum_{i=1}^{n} C_{11}^{i}\left(\frac{h_{i}^{3} \alpha_{i}^{2}}{12}+h_{i} \gamma_{i}^{2}\right) \\
\lambda_{3}=\sum_{i=1}^{n} C_{11}^{i} h_{i} \\
\lambda_{4}=\sum_{i=1}^{n} C_{11}^{i}\left(\frac{h_{i}^{3} \alpha_{i}^{2}}{12}+h_{i} \beta_{i} \gamma_{i}\right) \\
\lambda_{5}=\sum_{i=1}^{n} C_{11}^{i} h_{i} \beta_{i} \\
\lambda_{6}=\sum_{i=1}^{n} C_{11}^{i} h_{i} \gamma_{i} \\
\lambda_{37}=\sum_{i=1}^{n} C_{55}^{i} h_{i} \alpha_{i}^{2}
\end{gathered}
$$


where $C_{11}^{i}=\frac{E_{i}}{1-\nu_{i}^{2}}$ and $C_{55}^{i}=\frac{E_{i}}{2\left(1+\nu_{i}\right)}$.

The constants $\alpha_{i}, \beta_{i}$ and $\gamma_{i}$ are computed as follows:

For $i=1$,

$$
\left\{\begin{array}{l}
\alpha_{1} \\
\beta_{1} \\
\gamma_{1}
\end{array}\right\}=\left\{\begin{array}{l}
1 \\
0 \\
0
\end{array}\right\}
$$

For $i \geq 2$,

$$
\left\{\begin{array}{l}
\alpha_{i} \\
\beta_{i} \\
\gamma_{i}
\end{array}\right\}=\left\{\begin{array}{l}
N_{i}(2,2) \\
N_{i}(3,1) \\
N_{i}(3,2)
\end{array}\right\}
$$

where

$$
N_{i}=\left[\begin{array}{ccc}
1 & 0 & 0 \\
0 & B_{i} & 0 \\
C_{i} & F_{i} & 1
\end{array}\right] N_{i-1}
$$

with $N_{1}$ being the unit matrix and the constants $B_{i}, C_{i}$ and $F_{i}$ are defined as,

$$
\begin{gathered}
B_{i}=C_{55}^{i-1} / C_{55}^{i} \\
C_{i}=-\left(h_{i-1}+h_{i}\right) / 2 \\
F_{i}=-\left(h_{i-1}+A_{i} h_{i}\right) / 2
\end{gathered}
$$




\section{References}

[1] S. Subramanian, R. Surampudi, K. Thomson, S. Vallurupalli, Optimization of damping treatments for structure borne noise reductions, Sound Vib. 38 (2004) 14-19.

[2] M. D. Rao, Recent applications of viscoelastic damping for noise control in automobiles and commercial airplanes, J. Sound Vib. 262 (2003) 457474.

[3] E. Carrera, An assessment of mixed and classical theories on global and local response of multilayered orthotropic plates, Compos. Struct. 50 (2000) 183-198.

[4] E. Carrera, Theories and finite elements for multilayered, anisotropic, composite plates and shells, Arch. Comput. Methods Eng. 9 (2002) $87-140$.

[5] R. Mindlin, Influence of rotary inertia and shear on flexural motions of isotropic elastic plates, ASME J. Appl. Mech. 18 (1951).

[6] E. Reissner, The effect of transverse shear deformation on the bending of elastic plates, ASME J. Appl. Mech. 12 (1945) A69-A77.

[7] H. Hencky, Über die berücksichtigung der schubverzerrung in ebenen platten [on the introduction of shear motion in flat plates], Ing. Arch. 16 (1947) $72-76$.

[8] M. Levinson, An accurate, simple theory of the statics and dynamics of elastic plates, Mech. Res. Commun. 7 (1980) 343-350.

[9] J. N. Reddy, A simple higher-order theory for laminated composite plates, J. Appl. Mech. 51 (1984) 745-752.

[10] M. Karama, K. Afaq, S. Mistou, Mechanical behaviour of laminated composite beam by the new multi-layered laminated composite structures model with transverse shear stress continuity, Int. J. Solids Struct. 40 (2003) 1525-1546.

[11] X. Lu, D. Liu, Interlayer shear slip theory for cross-ply laminates with nonrigid interfaces, AIAA Journal 30 (1992) 1063-1073.

[12] C.-T. Sun, J. Whitney, Theories for the dynamic response of laminated plates, AIAA Journal 11 (1973) 178-183. 
[13] R. Ford, P. Lord, A. Walker, Sound transmission through sandwich constructions, J. Sound Vib. 5 (1967) 9-21.

[14] C. Smolenski, E. Krokosky, Dilational-mode sound transmission in sandwich panels, J. Acoust. Soc. Am. 54 (1973) 1449-1457.

[15] J. Moore, R. Lyon, Sound transmission loss characteristics of sandwich panel constructions, J. Acoust. Soc. Am. 89 (1991) 777-791.

[16] S. Narayanan, R. Shanbhag, Sound transmission through a damped sandwich panel, J. Sound Vib. 80 (1982) 315-327.

[17] S. Ghinet, N. Atalla, Modeling thick composite laminate and sandwich structures with linear viscoelastic damping, Comput. Struct. 89 (2011) $1547-1561$.

[18] S. Srinivas, A refined analysis of composite laminates, J. Sound Vib. 30 (1973) 495-507.

[19] J.-L. Guyader, C. Lesueur, Acoustic transmission through orthotropic multilayered plates, Part I: Plate vibration modes, J. Sound Vib. 58 (1978) 51-68.

[20] J.-L. Guyader, C. Lesueur, Acoustic transmission through orthotropic multilayered plates, part II: Transmission loss, J. Sound Vib. 58 (1978) 69-86.

[21] C. Lee, D. Liu, Layer reduction technique for composite laminate analysis, Comput. Struct. 44 (1992) 1305-1315.

[22] R. L. Woodcock, Free vibration of advanced anisotropic multilayered composites with arbitrary boundary conditions, J. Sound Vib. 312 (2008) 769-788.

[23] A. Loredo, A. Castel, A multilayer anisotropic plate model with warping functions for the study of vibrations reformulated from Woodcock's work, J. Sound Vib. 332 (2013) 102-125.

[24] A. Loredo, A multilayered plate theory with transverse shear and normal warping functions, Compos. Struct. 156 (2016) 361-374.

[25] E. Nilsson, A. Nilsson, Prediction and measurement of some dynamic properties of sandwich structures with honeycomb and foam cores, J. Sound Vib. 251 (2002) 409-430. 
[26] D. Backström, A. Nilsson, Modelling the vibration of sandwich beams using frequency-dependent parameters, J. Sound Vib. 300 (2007) 589611.

[27] D. Ross, E. E. Ungar, E. M. Kerwin Jr, Damping of plate flexural vibrations by means of viscoelastic laminae, Structural Damping 3 (1959) $44-87$.

[28] E. M. Kerwin Jr, Damping of flexural waves by a constrained viscoelastic layer, J. Acoust. Soc. Am. 31 (1959) 952-962.

[29] E. E. Ungar, E. M. Kerwin Jr, Loss factors of viscoelastic systems in terms of energy concepts, J. Acoust. Soc. Am. 34 (1962) 954-957.

[30] K. Ege, N. Roozen, Q. Leclere, R. Rinaldi, Assessment of the apparent bending stiffness and damping of multilayer plates; modelling and experiment, J. Sound Vib. 426 (2018) 129-149.

[31] G. Kurtze, B. G. Watters, New Wall Design for High Transmission Loss or High Damping, J. Acoust. Soc. Am. 31 (1959) 739-748.

[32] O. Zarraga, I. Sarría, J. García-Barruetabeña, F. Cortés, Homogenised formulation for plates with thick constrained viscoelastic core, Comput. Struct. 229 (2020) 106185.

[33] O. Zarraga, I. Sarría, J. García-Barruetabeña, F. Cortés, Dynamic analysis of plates with thick unconstrained layer damping, Eng. Struct. 201 (2019) 109809.

[34] C. Boutin, K. Viverge, Generalized plate model for highly contrasted laminates, Eur. J. Mech. A. Solids 55 (2016) 149-166.

[35] J.-L. Guyader, C. Cacciolati, Viscoelastic properties of single layer plate material equivalent to multi-layer composites plate, in: Turkish Acoustical Society - 36th International Congress and Exhibition on Noise Control Engineering, Inter-Noise 2007 Istanbul, volume 3, 2007, pp. 15581567.

[36] I. A. Viktorov, Rayleigh and Lamb waves, Plenum, New York, 1970.

[37] F. Marchetti, K. Ege, Q. Leclere, N. Roozen, On the structural dynamics of laminated composite plates and sandwich structures; a new perspective on damping identification, J. Sound Vib. 474 (2020) 115256. 
[38] D. L. Johnson, J. Koplik, R. Dashen, Theory of dynamic permeability and tortuosity in fluid-saturated porous media, J. Fluid Mech. 176 (1987) 379 .

[39] L. Jaouen, F. Chevillotte, Length Correction of 2D Discontinuities or Perforations at Large Wavelengths and for Linear Acoustics, Acta Acust. united Acust. 104 (2018) 243-250.

[40] F. J. Fahy, P. Gardonio, Sound and structural vibration: radiation, transmission and response, Elsevier, 2007.

[41] R. C. Hibbeler, Mechanics of Materials, Pearson, Boston, 2017.

[42] Q. Leclere, C. Pézerat, Vibration source identification using corrected finite difference schemes, J. Sound Vib. 331 (2012) 1366-1377. 